\title{
A multi-gap magnetorheological clutch with permanent magnet
}

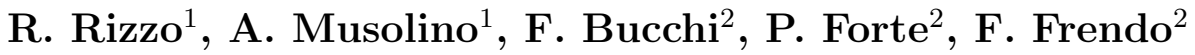 \\ ${ }^{1}$ Department of Energy and Systems Engineering, University of Pisa, Largo Lucio \\ Lazzarino, 56122, Pisa, Italy; \\ 2 Department of Civil and Industrial Engineering, University of Pisa, Largo Lucio \\ Lazzarino, 56122, Pisa, Italy. \\ E-mail: rocco.rizzo@unipi.it, antonino.musolino@unipi.it, \\ francesco.bucchi@for.unipi.it, p.forte@ing.unipi.it, \\ f.frendo@ing.unipi.it
}

\begin{abstract}
This paper describes the design and testing of a novel Permanent Magnet (PM) clutch based on Magneto-Rheological Fluid (MRF). It was inspired by a prototype previously developed by the authors and contains a novel gap shape conceived to reduce the torque loss in the disengaged operating mode. Several geometries and material arrangements were investigated and the performance in terms of transmissible torque in different operating conditions were assessed by FE numerical models. The prototype was manufactured and some experimental tests were performed. The new prototype was rated on the basis of performance indices and the design effectiveness was proved by a higher value of efficiency in the disengaged operating mode.
\end{abstract}

\section{Introduction}

Magnetorheological (MR) fluids are smart materials able to change their rheological properties. In few milliseconds [1] they pass from Newtonian-like fluids, if they are not subject to magnetic field, to viscoplastic-like fluids if subject to magnetic field. In magnetized conditions they exhibit a significant shear yield stress.

Their first applications date back to the 40's, but in the recent years they have been widely used in many fields thanks to the enhancements of their properties and to the development of magnetic finite element (FE) simulations. In particular, from the very beginning, MR fluids were studied for clutches and brakes. In 1948 Rabinow [2] designed the first magnetorheological clutch which was patented in 1951 [3], but for many years MR clutches did not find any applications due to the complexity of precisely assessing the magnetic flux within the fluid without the use of the FE method and the low quality of magnetorheological fluids. Recently many clutches and brakes have been designed and developed. In [4], the design and testing of a magnetorheological clutch with coils is described. Similarly, in [5] the design and testing of a magnetorheological 
brake is proposed. Many patents were produced on magnetorheological clutches and brakes $[6,7,8,9]$ which usually present plane, cylindrical, U-shaped or T-shaped gaps [10] and fluid excitation provided by coils.

The use of permanent magnets (PM) as a magnetic field source is not common. In [11] a possible geometry of clutch with PM (passive clutch) is presented, while in [12] a multi-gap clutch layout is proposed where the fluid magnetization can be obtained either with coils or with a permanent magnet. The design and testing of a fail-safe permanent magnet clutch was widely described by the authors in $[13,14,15]$ and the mechanical and magnetic models of the clutch were presented. The results were discussed in relation to simulations, showing a good agreement at high clutch slip whereas, at low slip, torque deviates from the one predicted [16] by the commonly used Bingham model [17].

In this paper a novel multi-gap magnetorheological clutch with permanent magnet is presented. The clutch design was conceived to disengage auxiliary devices in internal combustion engines. In particular, the specific application dealt with the disengagement of the vacuum pump which continuously draws air from the power-brake boost. Once the low pressure level in the boost have been reached, the vacuum pump operation is no longer necessary and, if it could be disconnected from the engine, consumption and pollution emission reduction would be achieved [18]. The main drawback of the previous design (old clutch) was related to the energy loss due to the Coulomb friction at the external seal when the clutch was disengaged. For this reason, the new prototype (new clutch) has a new gap shape and a different material and assembly arrangement, in order to reduce the power dissipated by the disengaged clutch. The material choice, the magnetic FE analysis and its validation, as well as the experimental testing are presented. In addition, the measured clutch characteristics, in terms of torque versus slip, are measured. In order to quantify the improvement of the proposed clutch design with respect to previous ones the performance indices, which were introduced in [19], are employed. Thanks to these indices, the comparison between the new and the old clutch architectures was possible and it is discussed in the paper.

\section{Background and new prototype conception}

A permanent magnet MR prototype was developed and investigated by the authors in the recent past. Its basic design scheme is shown in Fig. 1. It is composed of the primary and the secondary shafts, with a permanent magnet (PM) which can slide coaxially, thus varying its distance with respect to a single bell-shaped magnetorheological fluid gap. When the magnet is placed close to the fluid (rightward in Fig. 1) the clutch is engaged (ON state); if the magnet is moved leftward the magnetic field is shielded by the iron ring of the secondary shaft and the clutch is disengaged (OFF state). As described in $[13,20]$, the PM movement is obtained by a pneumatic actuator (not shown in figure), which imposes a proper pressure force to move the permanent magnet along its axis direction, performing an $\mathrm{ON} / \mathrm{OFF}$ operation strategy.

The larger seal turned out to be the main issue for the old prototype. The friction 


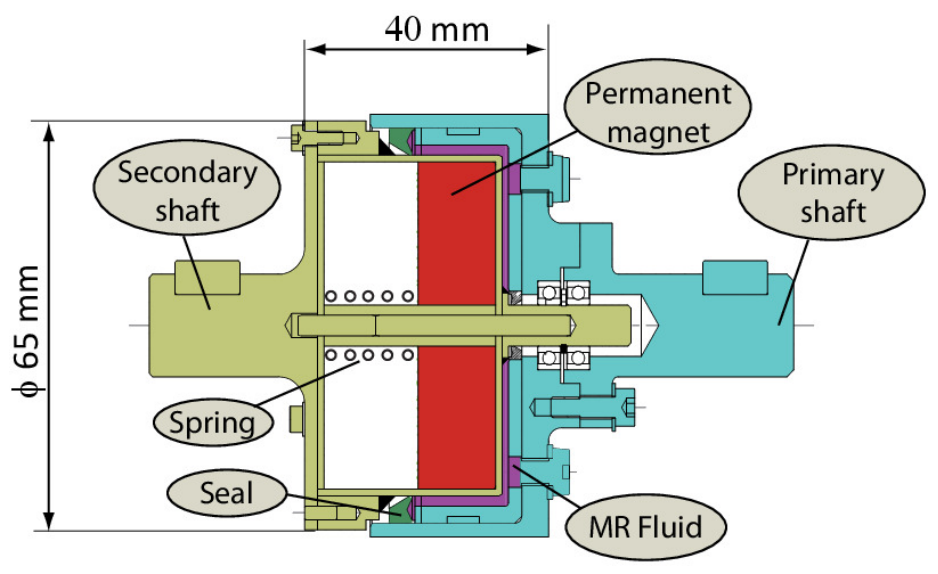

Figure 1. Previous clutch prototype scheme (old clutch).

losses were high due to the large distance from the axis of rotation. In addition, at high speed, due to the fluid centrifugation, some leakages were experienced in proximity of the larger seals, especially with unmagnetized fluid.

The basic idea which drove the design of the new prototype was to replace the external seal with a smaller one located at a smaller distance from the axis of the clutch to reduce the friction losses. For this reason, the novel gap shape schematically shown in Fig. 2 was conceived, with two cylindrical and two plane portions, which allows to place the sealing at the inner diameter.

The new gap shape was a challenging idea, which gave rise to a difficult mechanical and magnetic design phase aimed at developing a novel feasible device. The aim of the

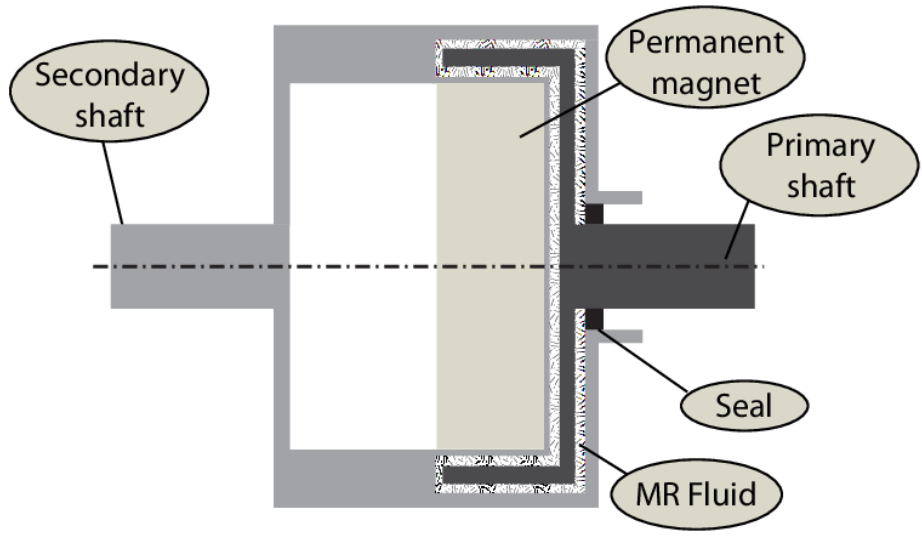

Figure 2. Geometry outline of the novel prototype (new clutch).

design was to maximize the magnetic flux density in the engaged configuration and to avoid fluid magnetization in the disengaged condition. A reference $5 \mathrm{Nm}$ torque was assumed for the design of the engaged clutch. 


\section{Numerical simulations for the design of the new prototype}

\subsection{Evaluation of the engaged and disengaged torque}

In order to carefully identify the ferromagnetic and non-ferromagnetic materials arrangement around the fluid and the permanent magnets characteristics, an in-depth investigation was performed. Several geometries and material arrangements were considered, with a specific attention to the primary shaft. However, taking into account that some PM configurations do not allow using an axis-symmetric formulation, and that the magnetic field inside the fluid is not uniformly distributed and its direction can vary from point to point, all the simulations were performed using a fully 3-D FE model based on the code [21]. The magnetic flux density vector in the MR fluid was computed using the FE mesh, shown in Fig. 3 which contains about $2.5 \times 10^{6}$ elements and takes about 120 minutes of CPU time on a Linux machine with $24 G B$ of RAM.

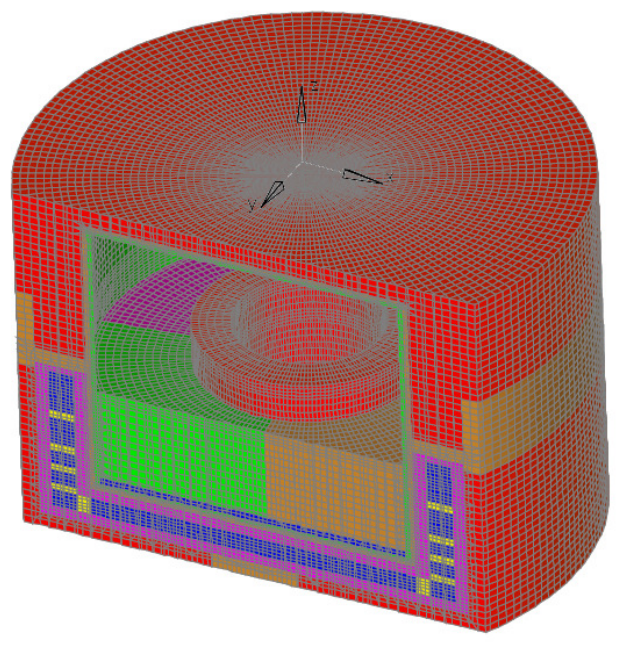

Figure 3. FE model of the device.

As a consequence, the geometric parameters of the clutch, that is the MRF gap dimensions, the material characteristics, as well as the permanent magnets configuration, were obtained at the end of an iterative procedure in which material choice and part assembly were driven by mechanical, technological and magnetic aspects.

The magnetic properties of AISI-1018 and AISI-316L were considered for the ferromagnetic and non-ferromagnetic parts respectively. The magnetic flux density vs. magnetic field characteristic ( $B-H$ curve) of the MRF140CG magnetorheological fluid produced by Lord Corporation was taken from the supplier data-sheet [22] and was imported in the simulations. Concerning the PM, as demonstrated in [14], for our application the highest MRF excitation level is obtained by using a PM system, composed of four $90^{\circ}$-poles, alternately magnetized along their diametral direction, (see Fig. 4), whose characteristics and dimensions are reported in Tab. 1. 


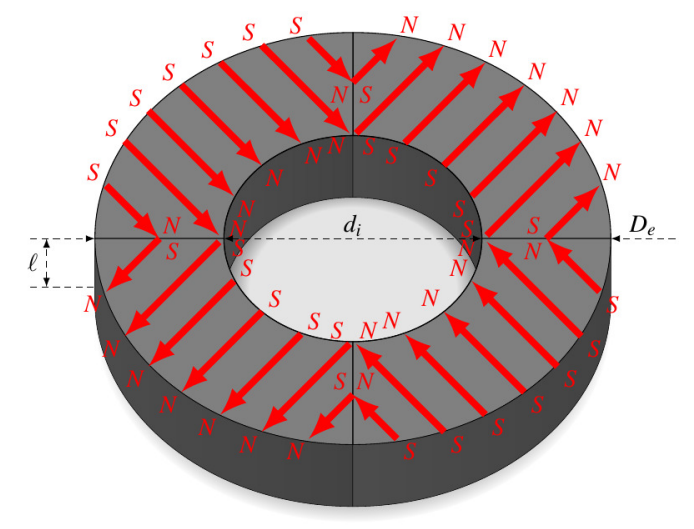

Figure 4. The permanent magnets system.

Table 1. PM exciter system characteristics and main dimensions.

\begin{tabular}{lll}
\hline Material & Physical Characteristics & Dimensions $(\mathrm{mm})$ \\
\hline \multirow{2}{*}{$\mathrm{NdFeB}$} & $B_{r}=1.36 \mathrm{~T} ;$ & $D_{e}=55.8( \pm 0.05) ;$ \\
& $H_{c}=10.2 \times 10^{5} \mathrm{~A} / \mathrm{m} ;$ & $d_{i}=30( \pm 0.05) ;$ \\
& $T_{\max }=150^{\circ} \mathrm{C} ;$ & $\ell=13( \pm 0.05) ;$ \\
\hline
\end{tabular}

Several geometries and material arrangements were considered, with a specific attention to the primary shaft. However, taking into account that some PM configurations do not allow using an axis symmetric formulation, and that the magnetic field inside the fluid is not uniformly distributed and its direction can vary from point to point, all the simulations were performed using a fully 3 D FE model based on the code [21]. The magnetic flux density vector in the MR fluid was computed using a FE mesh which eontains about $2.5 \times 10^{6}$ elements and takes about 120 minutes of CPU time on a Linux machine with $24 G B$ of RAM.

Figure 5 shows the magnetic flux density (vector and maps) in the MRF for two examined configurations of the primary shaft, when the clutch is in the engaged condition. Both configurations have the same dimensions but they are characterized by a different distribution of ferromagnetic and non-ferromagnetic material around the fluid gap. In particular, the main difference between configuration $\sharp 1$ and configuration $\sharp 2$ is the insertion, in the primary shaft of the latter one, of some non-ferromagnetic rings which allow to increase the magnetic flux density $B$ and improve its distribution in the MRF gap. Figure 5(a)-5(b) and Fig. 5(c)-5(d) respectively show the vector $B$ and its map in the fluid for the two configurations.

Figure 6 shows the magnetic flux density $B$ obtained in each part of the MR gap for the two examined configurations (in the figure, a sketched fluid gap helps the reader to see the line where the field was calculated). It can be observed that in many parts of the fluid gap, configuration $\sharp 2$ allows to obtain higher values of the field intensity.

The transmissible torque of the engaged $\left(T_{c}^{\mathrm{ON}}\right)$ and the disengaged $\left(T_{c}^{\mathrm{OFF}}\right)$ clutch was computed by using the magneto/mechanical model described in [14]. This 


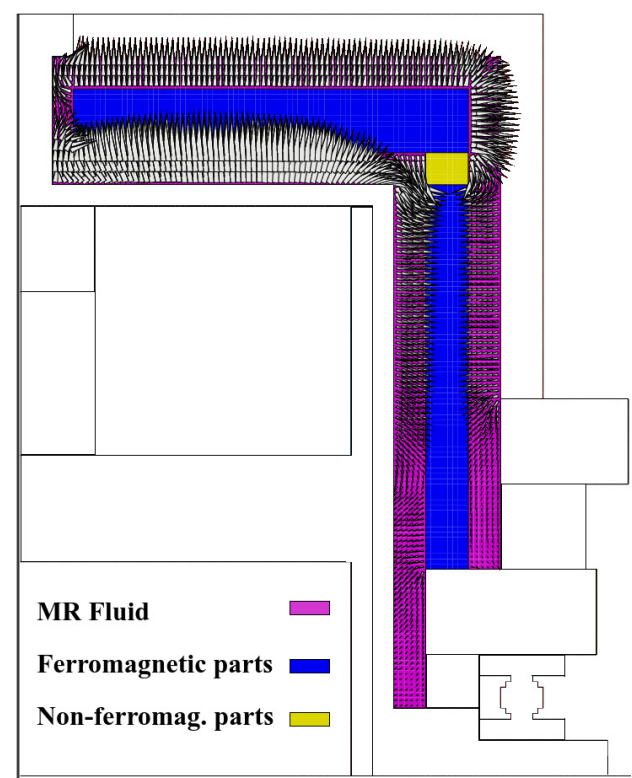

(a) configuration $\sharp 1$ : the vector $B$

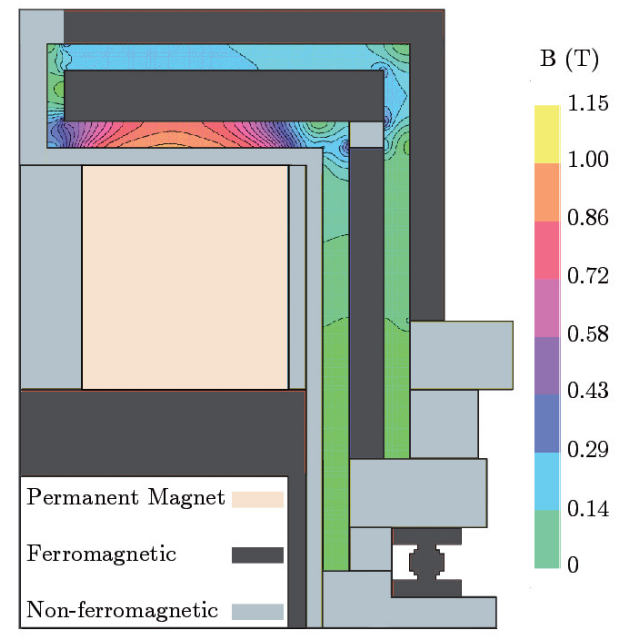

(c) configuration $\sharp 1$ : the map of $B$

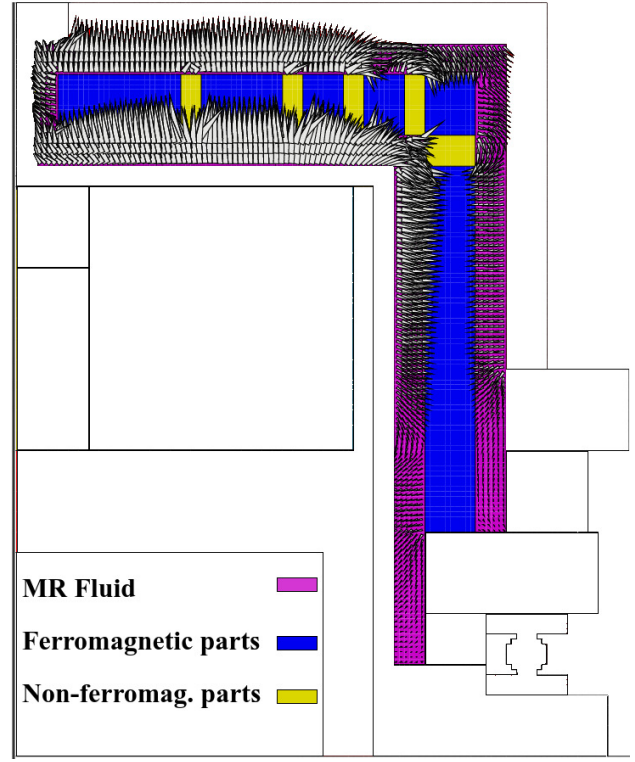

(b) configuration $\sharp 2$ : the vector $B$

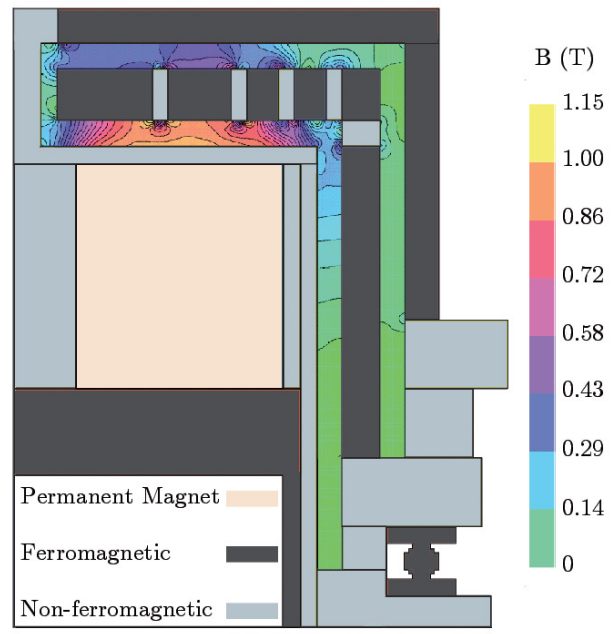

(d) configuration $\sharp 2$ : the map of $B$

Figure 5. Cross-section of the gap zone of the MR clutch. Example of magnetic simulations for two different configurations of the device.

mathematical model, developed on purpose and capable to exploit the 3D magnetic mesh of the FE code, allowed to obtain the transmissible torque by integrating the elementary contributions of the MRF cells, over the whole volume occupied by the fluid. The model was adapted to the new gap geometry, simply extending the integration domain. The results are listed in Tab. 2 and refer only to the torque due to the MR fluid magnetization, neglecting any viscous contribution.

A difference of $1.1 \mathrm{Nm}$ was assessed in the engaged configuration, while no significant difference emerged in the disengaged condition. For this reason, the configuration $\sharp 2$ was selected and further developed. 


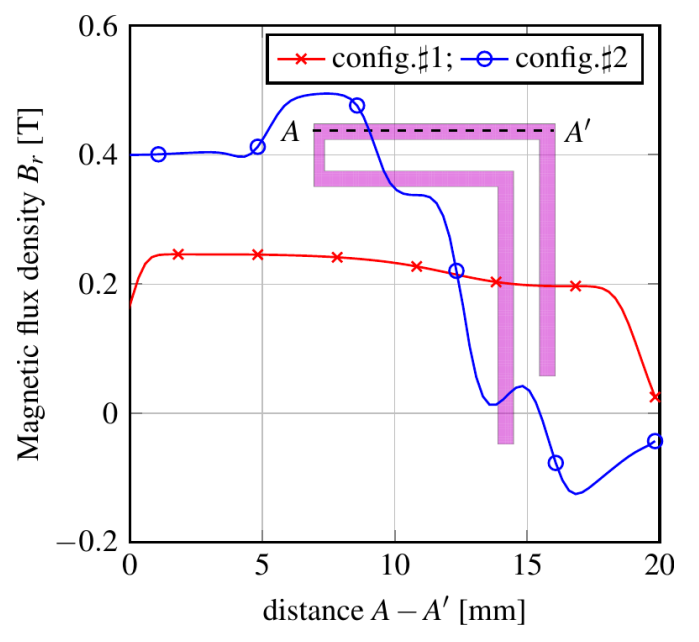

(a)

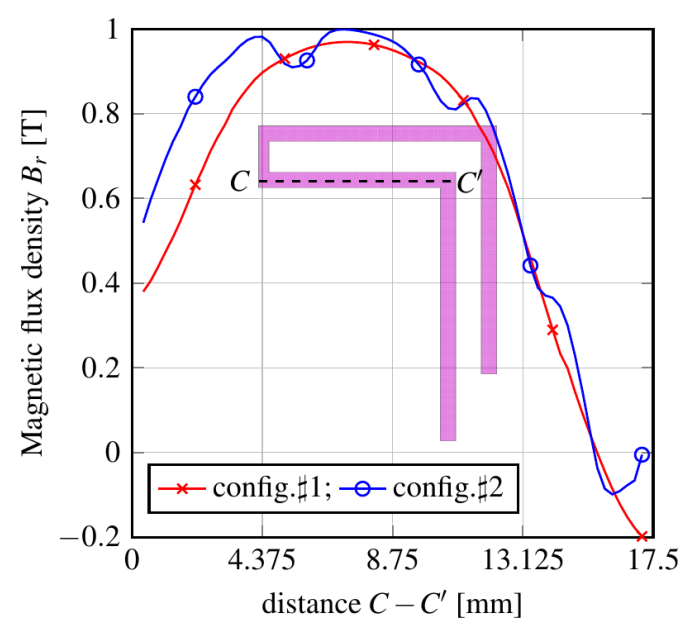

(c)

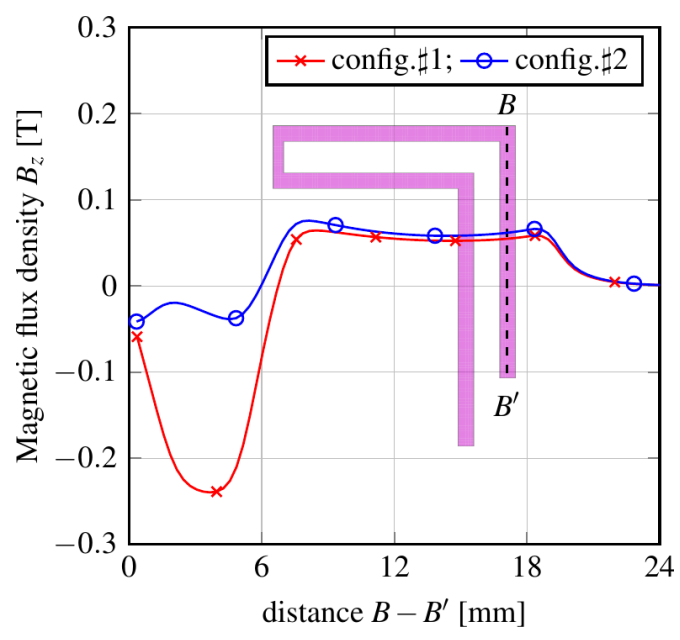

(b)

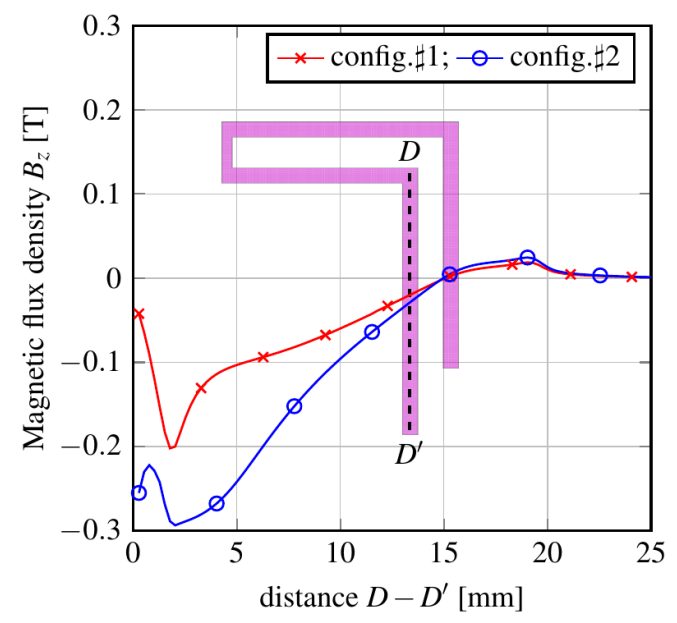

(d)

Figure 6. Comparison of the magnetic flux density $B$ in different parts of the fluids for the configuration $\sharp 1$ and the configuration $\sharp 2$. The dashed line shows the path where the field was measured.

Table 2. The computed torque values $([\Delta \omega]=\mathrm{rpm})$

\begin{tabular}{ccc}
\hline Configuration & $T_{\mathrm{c}}^{\mathrm{ON}}(\mathrm{Nm})$ & $T_{\mathrm{c}}^{\mathrm{OFF}}(\mathrm{Nm})$ \\
\hline$\sharp 1$ & $4.0+1.9 \times 10^{-4} \Delta \omega$ & $0.04+1.9 \times 10^{-4} \Delta \omega$ \\
$\sharp 2$ & $5.1+1.9 \times 10^{-4} \Delta \omega$ & $0.02+1.9 \times 10^{-4} \Delta \omega$ \\
\hline
\end{tabular}

\subsection{Dependence of the axial force and torque on the magnet position}

Even if the reference application is based on the ON/OFF control strategy in which the PM can be only placed in two positions (ON state or OFF state), the transmissible 
torque and the magnetic axial force were also computed as a function of the permanent magnet position. These curves, shown in Fig. 7, are useful to design the PM actuation system. From these results it follows that, the OFF state is characterized by very low magnetic flux density, resulting in a condition of disengaged shafts. On the contrary, during the PM movement from the $\mathrm{OFF}$ to the $\mathrm{ON}$ state, as the distance between the magnet itself and the fluid is reduced, the magnetic field inside the MRF increases. Then, when the PM is in the ON state the magnetic flux density is high enough to result in a high shear-stress and consequently in a high level of transmissible torque between the primary and secondary shaft. Taking into account that the simulations were performed in static conditions $(\Delta \omega=0)$, the values of the simulated torque in the two positions (ON state and OFF state) are in good agreement with the experimental data that will be shown further on in in figure 11.

For the clutch actuation, the axial force acting on the PM has to be considered. This force is due to the natural attractive behaviour between PM and ferromagnetic materials. Figure 7(b) shows the axial force on the PM, obtained by the FE simulation, as a function of its position. Since it may either hinder or facilitate the PM motion from the engaged state to the disengaged state and vice-versa, this magnetic axial force, added to the axial friction, should be taken into account in order to properly design the actuation system. As a preliminary experimental evaluation of the magnetic axial force, the rough but effective setup described in [14] was used. The measured magnetic axial forces, both in the engaged and disengaged conditions, differ from the simulated ones for an error below $5 \%$, showing a fully satisfactory agreement between them.

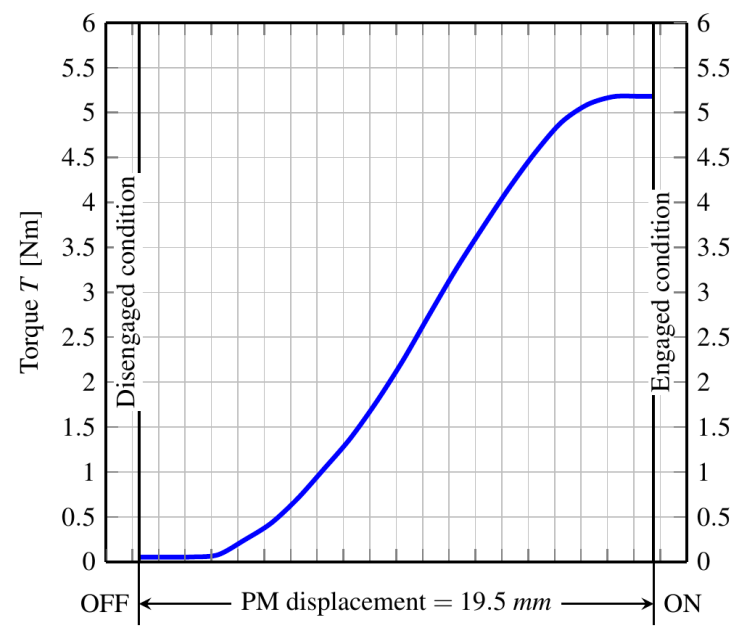

(a)

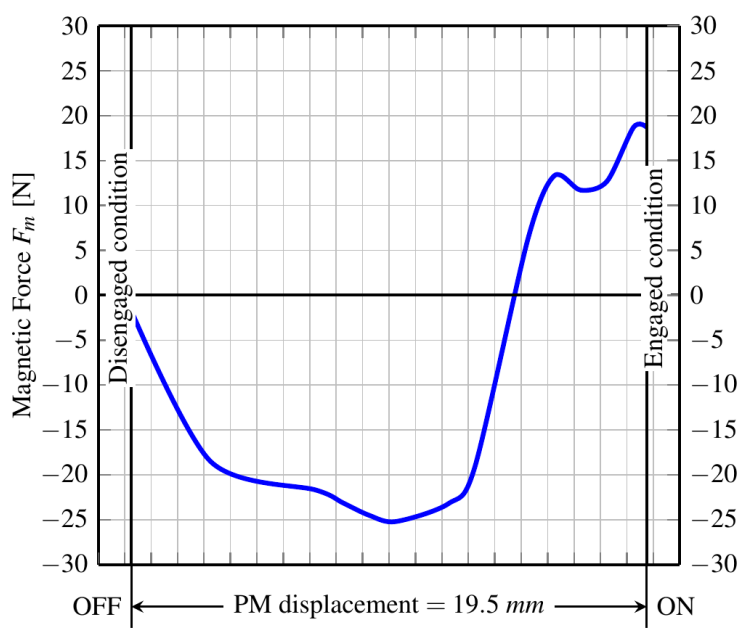

(b)

Figure 7. The transmissible torque (a) and the magnetic axial force (b) calculated by using the FEM model. 


\section{New prototype description}

The new device configuration with the novel gap geometry is shown in Fig. 8, with its main dimensions. The mass of the new prototype is $1.281 \mathrm{~kg}$ versus $0.864 \mathrm{~kg}$ of the old prope. The primary group is composed of a shaft fixed to a bell-shaped rotor with

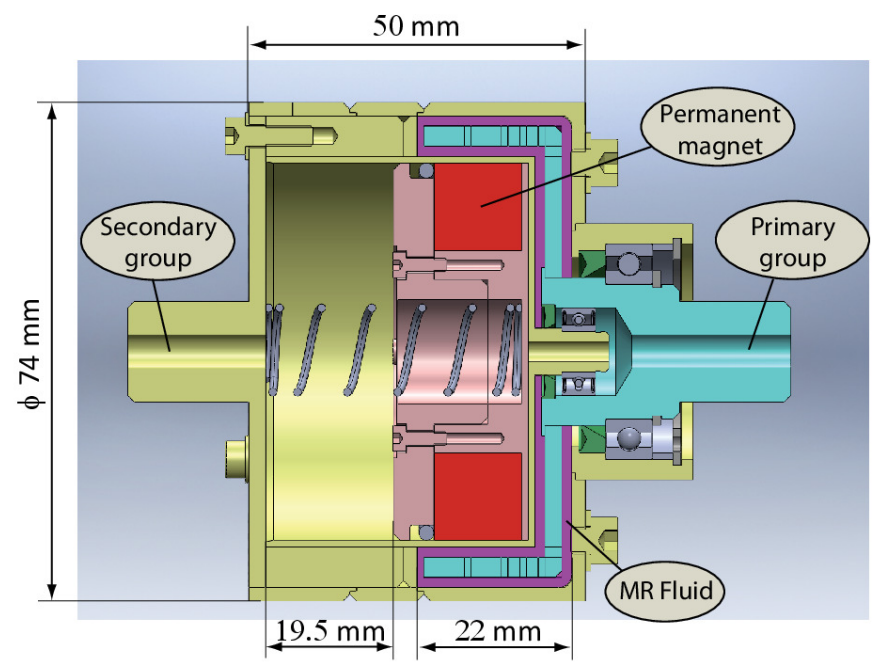

Figure 8. New prototype drawing.

alternated ferromagnetic and non-ferromagnetic parts, as resulted from simulations. The primary shaft is fixed to the bell-shaped part by welding, whereas the thin discs which form the bell are joined together by axial pins.

The secondary group has a more complex layout fulfilling external case and magnet housing functions. It is made up of three components made of ferromagnetic and non-ferromagnetic parts which are welded together. During the assembly, the magnet chamber can slide within the external case in order to assure the complete disassembly of the prototype. The rear cap is fixed to the magnet chamber by means of three screws.

The MR fluid is interposed between the primary and the secondary groups as in the old prototypes, but the gap geometry is more complex. Indeed, both the seals which confine the fluid are positioned at small diameters. Two holes are made axially in the front cover of the external case to fill the gap with the MR fluid. Primary and secondary parts are aligned by a couple of bearings, one of which also guarantees the axial position of the parts by means of two Seeger-rings. The ferromagnetic and non-ferromagnetic parts are made of AISI-1018 and AISI-316L, respectively.

The fluid magnetization is provided by the rare earth NdFeB hollow cylinder PM described in Section 3.1, which can slide axially in the housing chamber.

The basic operating principle is the same of the old prototype [13]: when the magnet is placed close to the fluid, as shown in Fig. 8, the clutch is engaged; if the magnet is moved leftward the clutch is disengaged.

As for the clutch actuation, although different actuators could be used (e.g. electrical 
solenoids, linear stepper motors, and so forth), a system based on a passive pneumatic operating principle was recognized as the most suitable for the specific application. A solution, which proved to be effective and reliable in the on/off control strategy, is described in details in $[13,20]$. The magnet, by means of an o-ring seal, splits the chamber in two hermetically separated parts. The primary and secondary shafts present an axial hole, which connects each part of the magnet chamber with an external pressure source. The operation principle of this solution is as follows: the engagement phase (moving the PM from the OFF to the ON state position) is assured by a preloaded spring; the disengagement phase can be obtained by the different pressures acting on the opposite magnet surfaces, which determines the shift of the PM system back to the OFF position. Anyhow, the actuation system was designed so that the spring forces the magnet toward the fluid gap zone; this condition guarantees the fluid magnetization in case of pneumatic system failure, assuring also a fail-safe clutch operation.

The prototype was manufactured and it is shown in Fig. 9. Its mass is $1.281 \mathrm{~kg}$ versus $0.864 \mathrm{~kg}$ of the old prototype. Additional manufacturing and assembly details are reported in [23].

Anyhow, considering that the new prototype is significantly different from the old one, in Section 6 a comparison between the new and the old prototypes is performed on the basis of non-dimensional performance indices, previously developed by the authors and described in [19]. They are able to compare the efficiency of the devices irrespective to their actual size and geometry.
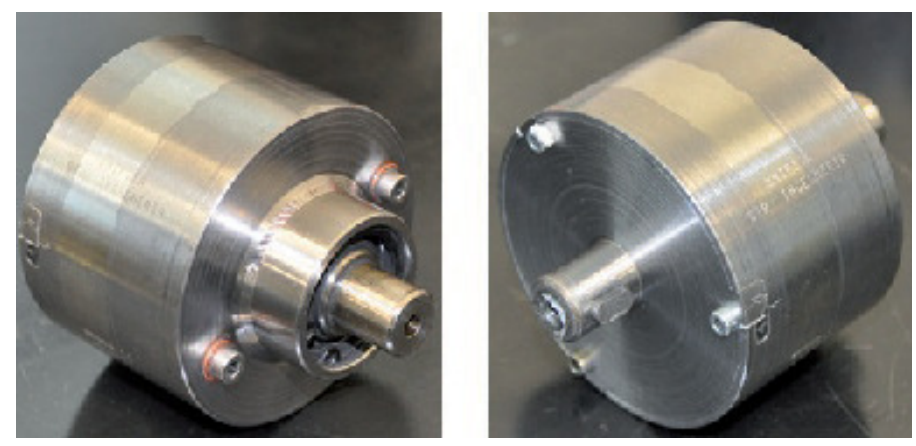

(a) Assembled prototype.

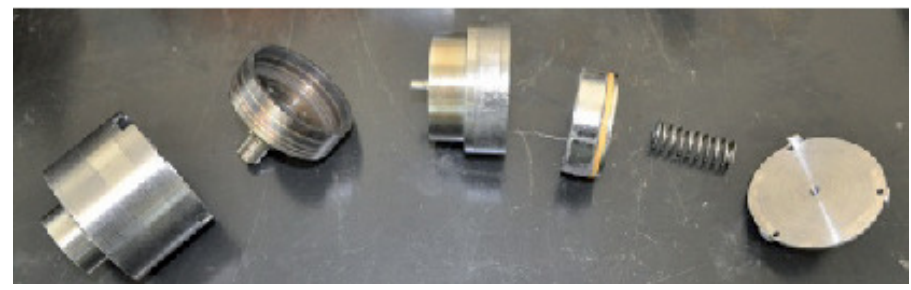

(b) Disassembled prototype: the external case, the multidisc rotor, the internal cylinder, the magnets system with shields and pneumatic o-ring, the spring and the rear cap (rightward order).

Figure 9. The manufactured prototype. 


\section{Experimental tests}

The new prototype was tested on the bench already described in [16]. The torque transmitted by the clutch versus the shaft speed difference $\Delta \omega$ was measured in four different states:

- without the MR fluid and with the PM removed from the clutch (Friction, $T^{\mathrm{F}}$ );

- with the gap filled and the PM removed from the clutch (Out, $T^{\mathrm{OUT}}$ );

- with the gap filled and the PM in the disengaged position (Disengaged, $T^{\mathrm{OFF}}$ );

- with the gap filled and the PM in engaged position (Engaged, $T^{\mathrm{ON}}$ );

Figure 10(a) shows the computed engaged torque $T_{\mathrm{c}}^{\mathrm{ON}}$ and the measured torque. The torque value is higher than $5 \mathrm{Nm}$ in the whole operating range and it keeps almost constant. Concerning the experimental results, some deviations from the ideal trend could be ascribed to secondary phenomena as the fluid heating and the effect of fluid rest time, analyzed by the authors in [24] and [25].

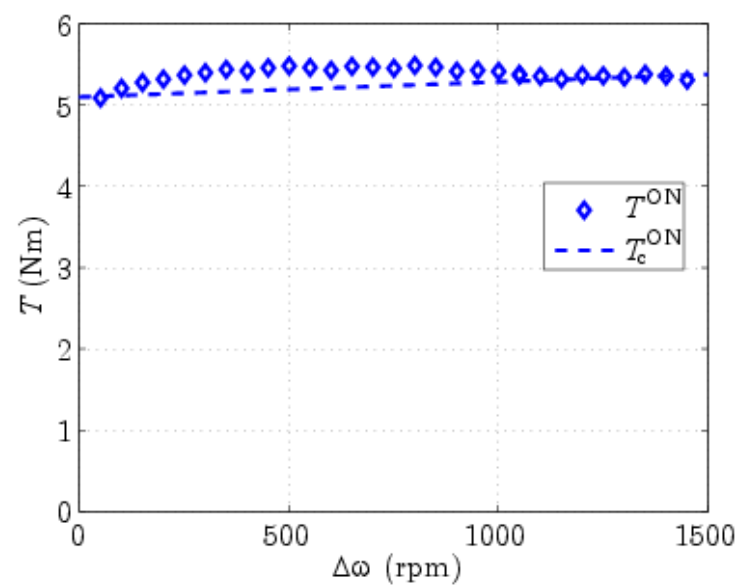

(a) engaged condition

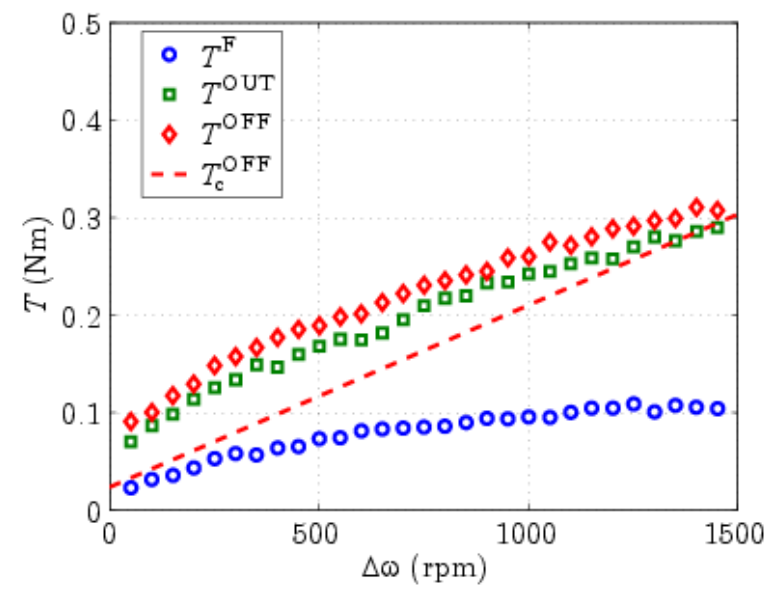

(b) disengaged condition

Figure 10. New prototype - Experimental characterization.

Concerning the non-magnetized fluid conditions, slight differences can be found between the three considered states (Friction, Out, Off). The difference between the $T^{\mathrm{OFF}}$ and $T^{\mathrm{OUT}}$ can be ascribed to the spurious fluid magnetization $\left(T_{\mathrm{c}}^{\mathrm{OFF}}\right.$ in Tab. 2). The observed small difference proves the effective magnet shielding in the disengaged state. The difference between the computed $\left(T_{\mathrm{c}}^{\mathrm{OFF}}\right)$ and measured $\left(T^{\mathrm{OFF}}\right)$ disengaged torque comes from the friction contribution and to the shear thinning phenomenon which occurs at high relative speed.

\section{Comparison of single and double-gap clutch prototypes}

A direct comparison of the torque of the old and new prototype is shown in Figs. 11(a)11(b). The engaged torque is strongly affected by the overall prototype size and it 


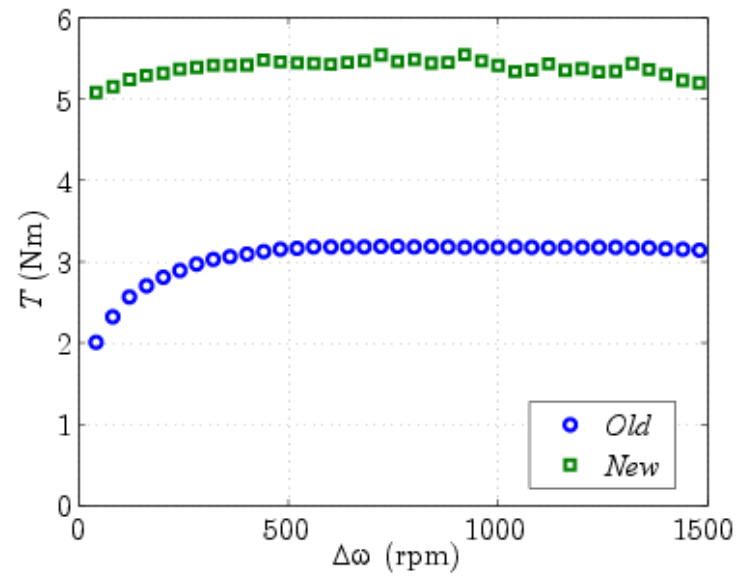

(a) engaged condition

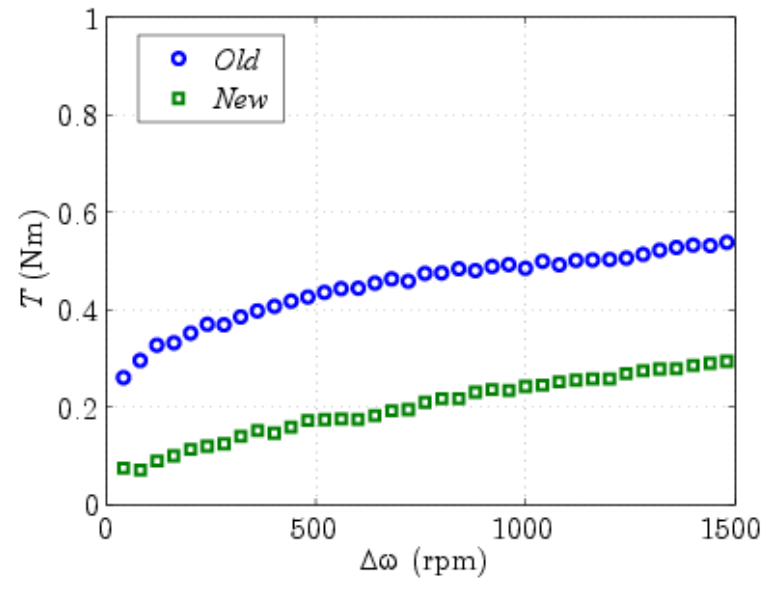

(b) disengaged condition

Figure 11. New prototype - Experimental characterization.

looks higher for the new (larger) prototype. On the contrary, despite the larger size, a considerable reduction of the disengaged torque was achieved for the new prototype. This was due to the reduction of friction and spurious fluid magnetization.

However the direct comparison of the absolute values of the torque in the $\mathrm{ON}$ and OFF state gives only limited indications about the effectiveness of the two prototype due to their different size and geometry. Then, in order to better compare the performance of the two different architectures, the non-dimensional indices introduced in [19] were used. The comparisen, in terms of performance, which can be achieved by the different architectures, can be obtained by the non-dimensional indices introduced in [19]. They were particularly considered to assess the performance of different clutches, despite the actual geometry and size. These indices are suitable to evaluate the degree of exploitation of the MR fluid in the ON state, and the effectiveness of the clutch design (friction and residual magnetization) in the OFF state, normalizing the values with reference values derived from the fluid properties and the device size. Indeed, they compare the actual engaged torque and the actual disengaged torque to two ideal reference torques, i.e. the maximum magnetorheological torque and the minimum dissipative torque which can be ideally achieved, respectively:

$$
\begin{aligned}
& \varepsilon^{\mathrm{ON}}(\Delta \omega)=\frac{T^{\mathrm{ON}}(\Delta \omega)-T^{\mathrm{OUT}}(\Delta \omega)}{T^{\mathrm{M}}} \\
& \varepsilon^{\mathrm{OFF}}(\Delta \omega)=\frac{T^{\mathrm{m}}(\Delta \omega)}{T^{\mathrm{OFF}}(\Delta \omega)}
\end{aligned}
$$

In the previous equations, $T^{\mathrm{ON}}(\Delta \omega)$ and $T^{\mathrm{OFF}}(\Delta \omega)$ are the experimentally measured torque in the engaged and disengaged states respectively, $T^{\mathrm{OUT}}(\Delta \omega)$ is the transmitted torque which is measured with the magnet removed from the clutch; $T^{\mathrm{M}}$ 


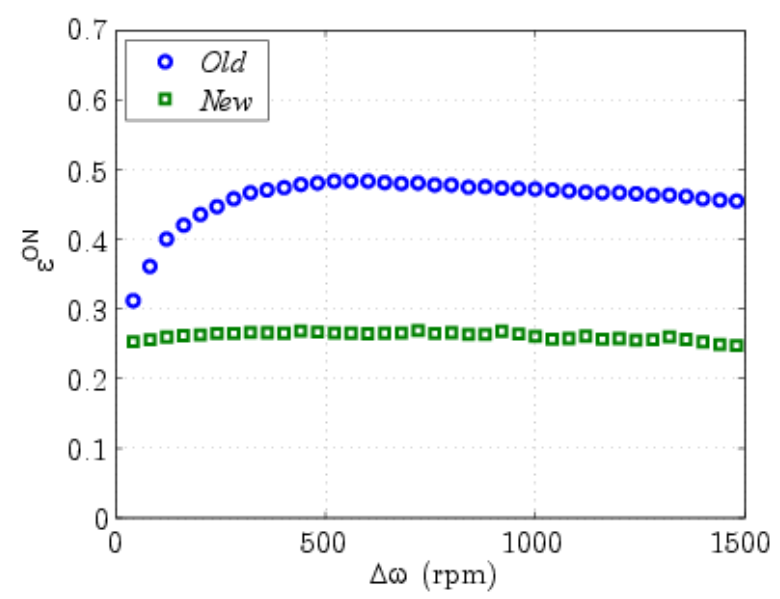

(a) Fluid exploitation index.

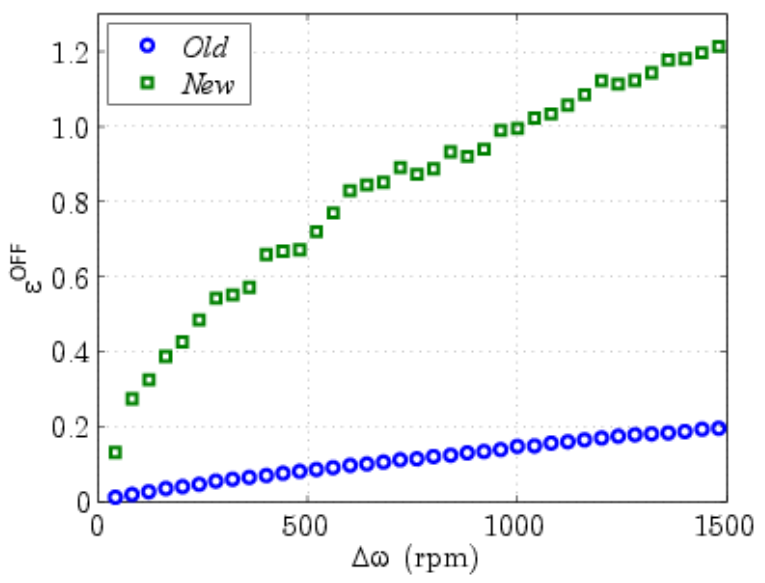

(b) Efficiency index.

Figure 12. Performance indices.

represents the ideal magnetorheological torque that can be obtained if the maximum shear stress $\tau^{M}$ is exploited throughout the whole gap and $T^{\mathrm{m}}(\Delta \omega)$ is the minimum torque due to the viscous stresses in the fluid, that is computed considering the viscosity of the unmagnetized MRF140CG fluid, and represents the torque ideally dissipated in the disengaged state in case of perfect shielding of the magnet and negligible friction losses. The use of such indices allows to compare the two devices efficiency irrespective to their actual size and geometry. In particular, the bigger the indices, the better the performance.

The performance indices were computed for the new prototype and compared to the ones obtained for the old prototype.

Concerning the fluid exploitation index (Fig. 12(a)) the new prototype is worse than the old one, due to the different geometry of the MR gap. Indeed, the magnetic flux density in the external cylindrical gap is weak and decreases the value of the index. On the contrary, the efficiency is very high, especially at high speed, where the shear thinning phenomenon appears, and the torque absorbed by the clutch is lower than the one predicted by the constant-viscosity model $\left(\varepsilon^{\mathrm{OFF}}>1\right)$. 


\section{Conclusions}

The design of a novel PM MR clutch prototype was presented in this paper. Its study was motivated by the wish to settle the drawbacks of a MR prototype previously developed by the authors. In particular a novel gap shape was conceived in order to reduce the torque friction losses of the disengaged clutch.

Several geometries and material arrangements were considered and their torque performance was assessed by FE numerical models. Once the final geometry was identified, a clutch prototype was manufactured and tested. The experimental tests were aimed at measuring the torque transmitted in the engaged and disengaged states and the axial force acting on the PM. The results were in agreement with the simulations and proved the effectiveness of the numerical models.

Finally, a comparison between the new and the old prototype was proposed. Since the comparison in terms of transmitted torque is strongly affected by the prototype size and geometry, the performance indices, introduced by the authors in [19], were employed. By this analysis, as expected, the fluid exploitation was lower for the new prototype, since the external gap is subject to low magnetic flux density. On the contrary, the new clutch prototype proved to be much more efficient in the disengaged state, verifying the effectiveness of the design improvements.

\section{Acknowledgments}

This work was funded by Pierburg Pump Technology Italy, S.p.A., within a framework of a project supported by Regione Toscana, P.O.R., C.R.e.O., F.E.S.R. 2007-2013.

\section{References}

[1] A. G. Olabi and A. Grunwald, "Design and application of magneto-rheological fluid," Materials E3 Design, vol. 28, no. 10, pp. 2658-2664, 2007.

[2] J. Rabinow, "The magnetic fluid clutch," AIEE Transactions, pp. 1308-1315, 1948.

[3] J. Rabinow, "Magnetic fluid torque and force transmitting device," U.S. Patent, p. 2575360, 1951.

[4] B. M. Kavlicoglu, F. Gordaninejad, C. A. Evrensel, N. Cobanoglu, Y. Liu, A. Fuchs, and G. Korol, "High-torque magnetorheological fluid clutch," in SPIE's 9th Annual International Symposium on Smart Structures and Materials, pp. 393-400, International Society for Optics and Photonics.

[5] W. H. Li and H. Du, "Design and experimental evaluation of a magnetorheological brake," International Journal of Advanced Manufacturing Technology, vol. 21, pp. 508-515, 2003.

[6] S. Gopalswamy, S. M. Linzell, and G. L. Jones, "Magnetorheological fluid clutch with minimized reluctance," U.S. Patent, p. 5845752, 1998.

[7] P. B. Usoro, A. L. Smith, C. K. Kao, M. George, and G. Sommer, "Magnetorheological fluid clutch," U.S. Patent, p. 6318531, 2001.

[8] E. A. Bansbach, "Torque transfer apparatus using magnetorheological fluids," U.S. Patent, p. $5779013,1998$.

[9] J. D. Carlson, "Magnetorheological brake with integrated flywheel," U.S. Patent, p. 6186290, 2001. 
[10] Q. H. Nguyen and S. B. Choi, "Selection of magnetorheological brake types via optimal design considering maximum torque and constrained volume," Smart Materials and Structures, vol. 21, no. 1, p. 015012, 2012.

[11] G. L. Johnston, W. C. Kruckemeyer, and R. E. Longhouse, "Passive magnetorheological clutch," U.S. Patent, p. 5848678, 1998.

[12] H. Steinwender, "Magnetorheological clutch," U.S. Patent, p. 7588131, 2009.

[13] F. Bucchi, P. Forte, F. Frendo, R. Rizzo, and A. Musolino, "A fail-safe magnetorheological clutch excited by permanent magnets for the disengagement of automotive auxiliaries," Journal of Intelligent Material Systems and Structures, vol. 25, no. 16, pp. 2102-2114, 2014.

[14] R. Rizzo, A. Musolino, F. Bucchi, P. Forte, and F. Frendo, "Magnetic FEM design and experimental validation of an innovative fail-safe magnetorheological clutch excited by permanent magnets," IEEE Trans. on Energy Conv., vol. 29, no. 3, pp. 628-640, 2014.

[15] G. Armenio, E. Bartalesi, F. Bucchi, A. Ferri, F. Frendo, P. Forte, R. Rizzo, and R. Squarcini, "Mechanical combustion engine driven fluid pump.," 2011.

[16] F. Bucchi, P. Forte, and F. Frendo, "Experimental characterization of a permanent magnet magnetorheological clutch," ASME 2012 11th Biennial Conference on Engineering Systems Design and Analysis, vol. 4, pp. 345-355, 2012.

[17] E. C. Bingham, An investigation of the laws of plastic flow, vol. 13 of Bulletin of the Bureau of Standards. Govt. Print. Off., 1917.

[18] F. Bucchi, P. Forte, F. Frendo, and R. Squarcini, "A magnetorheological clutch for efficient automotive auxiliary device actuation.," Fratt. e Integr. Strutt., vol. 23, pp. 62-74, 2013.

[19] F. Bucchi, P. Forte, A. Franceschini, and F. Frendo, "Analysis of differently sized prototypes of an MR clutch by performance indices," Smart Mater. and Struct., vol. 22, no. 10, p. 105009, 2013.

[20] E. Bartalesi, F. Bucchi, and R. Squarcini, "Vacuum actuation for axial movement of a magnet in a magnetorheological clutch," EPO Patent Pending, 2012.

[21] EFFE, "EFFE v2.00, User Manual," Bathwick Electrical Design ltd, 2009.

[22] L. C. Ltd., "http://www.lord.com/."

[23] F. Bucchi, "Smart materials and vehicle efficiency. Design and experimentation of new devices.," PhD Thesis - University of Pisa, 2014.

[24] F. Bucchi, P. Forte, and F. Frendo, "Temperature effect on the torque characteristics of a magnetorheological clutch," Mechanics of Advanced Materials and Structures, vol. 22, no. 12, pp. 150-158, 2015.

[25] F. Bucchi, P. Forte, and F. Frendo, "Analysis of the torque characteristic of a magnetorheological clutch using neural networks," Journal of Intelligent Material Systems and Structures, p. 1045389X14546654, 2014. 